\title{
Is it Photocatalytic Degradation of Textile Dyes a Friendly Method? Methyl Violet Dye as a Model for Application in Aqueous Solutions in the Presence of Commercial $\mathrm{TiO}_{2}$
}

\author{
Falah H. Hussein, Aseel M.Ajobree, Zeyad O. Musa, Firas H. Abdulrazzak, Mohammed Bassim \\ Alqaragoly, Ayad F. Alkaim
}

\begin{abstract}
In this study, we evaluated the photocatalytic activities of analytical reagent $(A R)$ grade TiO2 to identify a low-cost photocatalyst for dye degradation.studied different conditions in the presence of TiO2 suspension. The effectof various parameters such as mass of titanium dioxide, dye concentrationon the photocatalytic degradation were investigated. Results showed that, thephotocatalytic degradation process was high at the beginning and then decreased with time. This process was well described by pseudo, first order kinetics according to the Langmuir-Hinshelwood model.

The results explained the photocatalytic degradation efficiency, which was increased by increasing catalyst loading from0.05 to $0.3 \mathrm{~g} / 100 \mathrm{ml}$. Results also showed that the rate of photocatalytic degradation was increased with decreasing dye concentration.
\end{abstract}

Keywords : Photocatalytic degradation, Titanium dioxide, Methyl violet, Light intensity.

\section{INTRODUCTION}

Dyes colored, ionizing and aromatic organic compounds which show an affinity towards the substrate to which it is being applied. It is generally applied in aqueous solution. Dyes may also require a mordant to better the fastness of the dye on the material on which it is applied [1-4]. Dyes may be classified in several ways, according to their chemical constitution, application, origin and use. They can be natural and synthetic based on their origin. Dyes are classified into acidic, basic, mordant, direct, reactive, vat, disperse, sulfur, azoetc based on their applications. [5-8]

Revised Manuscript Received on July 22, 2019.

Falah H. Hussein, Aseel M.Ajobree, Zeyad O. Musa, Firas H. Abdulrazzak, Mohammed Bassim Alqaragoly, Ayad F. Alkaim

Almustaqbal University College, HILLA, IRAQ

College of science for women-Chemistry Department/ University of Babylon -Iraq

College of Education for Pure Sciences -Chemistry Department /Diyala University-Iraq.

Alhilla college University/Iraq
Dyes and pigments are extensively used in industries that manufacture and process textiles, paper, plastics, leather, food, and cosmetic products. The release of dye-containing effluents from these industries into water bodies poses a considerable threat to aquatic life as well as the environment. Numerous studies have investigated the efficient removal of hazardous organic molecules from waste water during past decades. These studies have demonstrated that the hazardous organic molecules in waste water can be degraded using photo excited charge carriers in an excited semiconductor [9]semiconductors are wide-band-gap semiconductors, which require ultraviolet (UV) irradiation for photocatalysis When a wide-band-gap photocatalyst is irradiated with light of energy equal to or higher than its band-gap energy, electron-hole pairs are created. In an aqueous medium, reactants may be adsorbed on the surface of a photocatalyst and may react directly or indirectly with the photo-generated electrons and holes .

\section{EXPERIMENTAL PART}

\section{A. Materials}

Commercial $\mathrm{TiO} 2$ powder were purchased from (sigma-aldrich, Germany) Methyl Violet dye (MV) was supported by Hilla Company of textile dyes/ Iraq. All chemicals used in this study were analytical grade and used directly without further purification. A stock solution of $1000 \mathrm{mg} / \mathrm{L}$ was prepared by dissolving (1 $\mathrm{gm})$ as an appropriate quantity of textile dye (MV) in (1 L) double distilled water.

Photo catalytic experiments

Photocatalytic degradation was carried out in an experimental setup consist of a homemade photoreactor. Irradiation sourceis Philips mercury lamp UV (A), contains six lamps of $15 \mathrm{~W}$ for each one (Germany). Most experiments were 
carried out in a reactor containing $400 \mathrm{~cm} 3$. The lamp was positioned perpendicularly above the radiation vessel and the distance was fixed for a chosen light intensity. In all experiments, the required amount of the catalyst was suspended in $200 \mathrm{~cm} 3$ of aqueous solutions of dye, by using a magnetic stirrer. At predominant times, 3 $\mathrm{cm} 3$ of reaction mixture was collected and centrifuged (6000 rpm, 10 minutes) in a Hettich centrifuge. The supernatant was carefully collected in a syringe with a long pliable needleand centrifuged for a second time, at the same speed and for the same period of time. This second centrifugation was found necessary to remove the fine particles of $\mathrm{TiO} 2$ that found effective on the result of analysis by UV-Visible spectrophotometer. dye concentration was determined by Parkash et al.8 at a wavelength $580 \mathrm{~nm}$, by using UV-Visible spectrophotometer (Type Apel PD-303 UV). All experiments carried outunder temperature $298 \mathrm{~K}$.

\section{RESULT AND DESICCATION}

\section{Effect of mass dosage}

A $15 \mathrm{mg} / \mathrm{l}$ aqueous solution of methyl violet was used in this study. About $100 \mathrm{ml}$ of solution with catalyst amount of $0.05 \mathrm{~g} / 100 \mathrm{ml}$ to $0.3 \mathrm{~g} / 100 \mathrm{ml}$ was added. Before irradiation the system was magnetically stirred for 15 minutes under dark to establish the adsorption-desorption equilibrium between the catalytic surface and the dye. The irradiation time was limited to one hour based on the lamp life. The absorbance of the solution before and after the irradiation was measured using spectrophotometer at $580 \mathrm{~nm}$. The profile behavior of photocatalytic degradation is illustrated in Fig. 1.

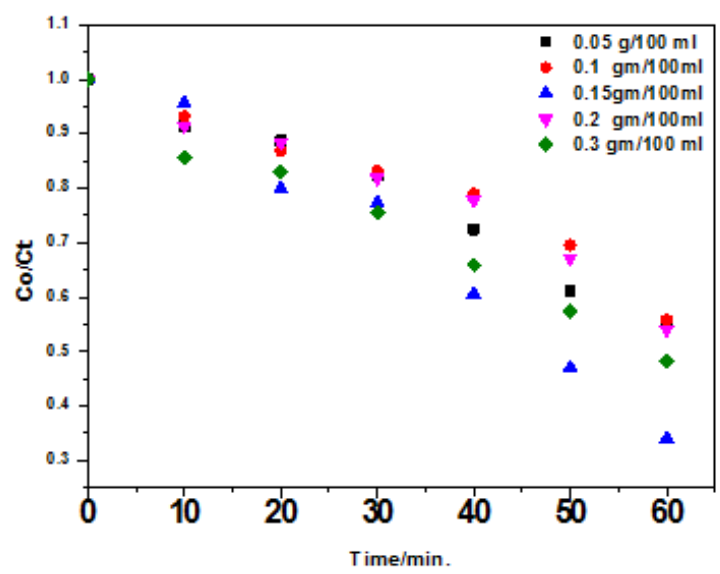

Fig.1: Photocatalytic degradation of MV dye at different mass dosage. Experimental

Conditions: Initial conc. $15 \mathrm{mg} . \mathrm{L}^{-1}$, Temp. $30{ }^{\circ} \mathrm{C}$, and L.I. $2.42 \mathrm{~mW} . \mathrm{cm}^{-2}$.
Fig.2 represents the percent degradation of Methyl violet against the different amount of catalyst. This shows that the percent degradation of modified catalysts increase with increase in the amount of catalyst from $0.05-0.3$ $\mathrm{g} / 100 \mathrm{ml}$ and above this limit there is not much change.[10-12]. This indicated that the active site provided for the adsorption of substrate on the catalyst surface is limited to catalyst amount of $0.15 \mathrm{~g} / 100 \mathrm{ml}$ and after that no much change in the degradation. At higher dosage the vacant sites are consumed by the intermediate products obtained during the reactions which retard further degradation of the substrate. Hence the percent degradation decreased or retained without a noticeable change [13-17].

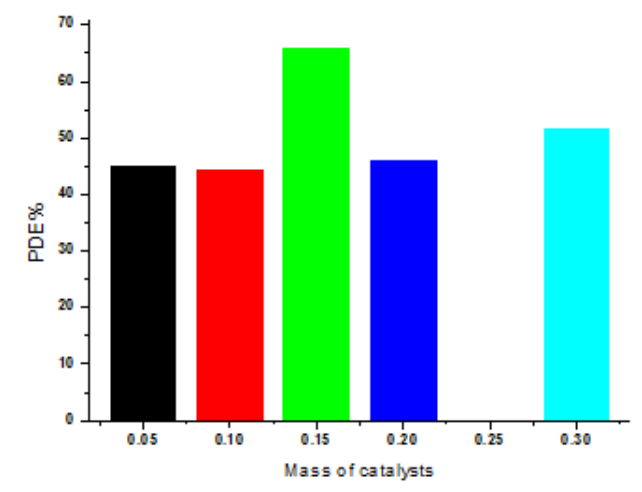

Fig.2 photo catalytic degradation efficiencyunder different catalyst loading. Experimental Conditions: Initial conc. $15 \mathrm{mg} . \mathrm{L}^{-1}$, Temp. $30{ }^{\circ} \mathrm{C}$, and L.I. 2.42 $\mathrm{mW} \cdot \mathrm{cm}^{-2}$.

\section{EFFECT OF CONCENTRATION OF DYE}

The effect of MV dye concentration has been investigated at $\mathrm{pH}$ 6.2, catalyst concentration 0.15 $\mathrm{g} / 100 \mathrm{ml}$, light intensity L.I. $2.42 \mathrm{~mW} . \mathrm{cm}^{-2}$, flow rate of $\mathrm{O} 2\left(5 \mathrm{~mL} \cdot \mathrm{min}^{-1}\right)$ and $\mathrm{MV}$ dye concentrations (5-25 $\mathrm{mg} / \mathrm{L})$. [11, 18-20] .The experimental data could be analyzed to assume-first order kinetic as shown in Figure 3.

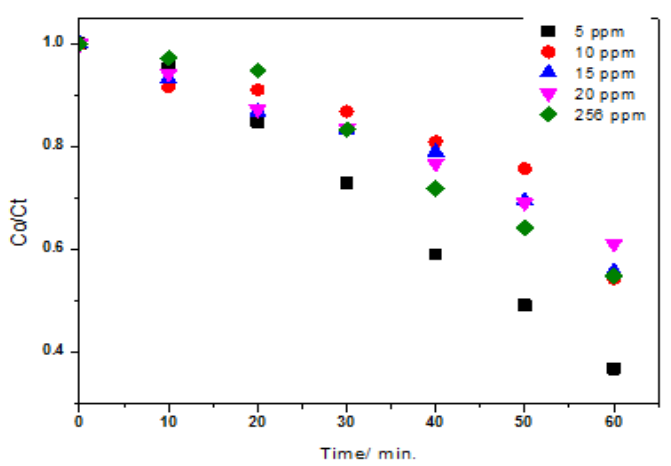

Fig.3: Photo catalytic degradation of MV dye at different initial concentration. 
Experimental Conditions: mass amount 1.5 g.L ${ }^{-1}$, Temp. $30{ }^{\circ} \mathrm{C}$, and L.I. $2.42 \mathrm{~mW} . \mathrm{cm}^{-2}$.

The excess of MV dye prevent the penetration of light through the successive layers of $\mathrm{MV}$ on the $\mathrm{TiO}_{2}$ surface is weak to generate the required excited state of the MV dye on $\mathrm{TiO}_{2}[21][22]$. The concentration of $\mathrm{MV}$ dye 5 ppm gives the optimum Photocatalytic degradation efficiency which is equal to64\%. The results of the change in percentage Photocatalytic degradation efficiency (P.D.E \%) with concentration of MV dyeplotted in Figure 4.

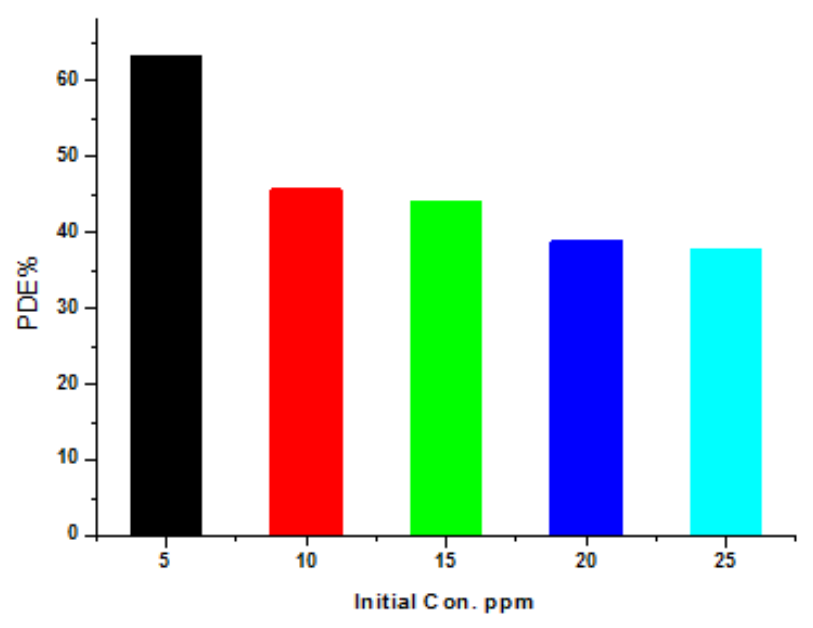

Fig. 4. Effect of initial dye concentration on the photo catalytic degradation of MV dye. Experimental Conditions: mass amount 1.5 g. $\mathrm{L}^{-1}$, Temp. $30{ }^{\circ} \mathrm{C}$, and L.I. $2.42 \mathrm{~mW} . \mathrm{cm}^{-2}$.

\section{CONCLUSIONS}

The obtained results show that MV can be easily degraded by a $\mathrm{TiO}_{2}$ assisted process in aqueous dispersions under UV irradiation. The photo degradation of the dye follows first order kinetics and parameters like the Effect of mass dosageTiO ${ }_{2}$ and Effect of concentration of dye on the photo degradation.The results indicated that the catalyst loading, the initial dye concentration affected the degradation efficiency of $\mathrm{TiO}_{2}$ powders obviously. We learned that the photo degradation efficiency is enhanced with the increase of catalyst loading and the reverse effect is obtained with the increase of initial dye concentration in our experiments. The photo catalytic decomposition of MV dye was most efficient in the solution at lower initial concentration which indicate our method more friendly and economically.

\section{REFERENCES}

1. Aseel M Aljeboree, A.N.A., Ayad F Alkaim, Kinetics and equilibrium study for the adsorption of textile dyes on coconut shell activated carbon. Arabian journal of chemistry, 2017. 10: p. 3381-3393.

2. Y.mat., h.y.j.o.a.t.y.x.x.1.x.d.y., resr. bulln., 2006. 41: p. 1310.

3. Alqaraguly, a.f.a.a.m.b., adsorption of basic yellow dye from aqueous solutions by activated carbon derived from waste apricot stones (asac): equilibrium, and thermodynamic aspects. Int. J. Chem. Sci., 2013. 11(2 ): p. 797-814.

4. Aseel M Aljeboree, A.F.A., Ammar H Al-Dujaili, Adsorption isotherm, kinetic modeling and thermodynamics of crystal violet dye on coconut husk-based activated carbon. Desalination and Water Treatment, 2015. 53(13): p. 3656-3667.

5. Aljeboree, A.M., Adsorption of methylene blue dye by using modified Fe/Attapulgite clay Research Journal of Pharmaceutical, Biological and Chemical Sciences, 2015. 6(4): p. 778.

6. Alkaim, A.F., Alrobayi, Enas M ,Algubili, Abrar M and Aljeboree, Aseel M, Synthesis, characterization, and photocatalytic activity of sonochemical/hydration-dehydration prepared $\mathrm{ZnO}$ rod-like architecture nano/microstructures assisted by a biotemplate. Environmental technology, 2017. 38(17): p. 2119-2129.

7. E., A.V.B.C.B.P.A.G.-L.E.L.V.M.S.M.G.P.L.P.M.P., Chemosphere, 2002. 49: p. 1223.

8. Aljeboree, A.M., Adsorption of crystal violet dye by Fugas Sawdust from aqueous solution. International Journal of ChemTech Research, 2016. 9: p. 412-423.

9. Wang, C., Zhang, Y., Zhu, T., Wang, P., and Gao, S., Photocatalytic degradation of methylene blue and methyl orange in a Zn(II)- based Metal-Organic Framework. Desalination Water Treat, 2016. 57: p. 17844-17851.

10. H. Wang, C. Xie, W. Zhang, S. Cai, Z. Yang, and Y. Gui, Comparison of dye degradation efficiency using $\mathrm{ZnO}$ powders with various size scales. J. Hazard Mater., 2007. 141(3): p. 645-652.

11. J. Kaur, and S. Singhal, Heterogeneous photocatalytic degradation of rose bengal: Effect of operational parameters. Physica B, 2014. 450: p. 49-53.

12. M. S. Mashkour, A. F. Alkaim, L. M. Ahmed, and F. H. Hussein, Zinc oxide assisted photocatalytic decolorization of reactive red 2 dye. Int. J. Chem. Sc., 2011. 9(3): p. 969-979.

13. M., G.M.S.T.O.-C.A.M.F.P.E.M.P.P.M.S.Q., J., Chemosphere 1999. 39: p. 781.

14. G., C.F.X.Y.Z.J.L., Chemosphere 2000. 44: p. 1159.

15. I., P.I.A., J. Chem. Technol. Biotechnol., 1999. 74 p. 349.

16. H., H.L.E.P.A.H.M.K.E.E.C.G.J., Appl. Catal. B: Environ., 2002. 39 p. 75.

17. T. Sauer, G. C. Neto, H.J. José, and R.F. Moreira, Kinetics of photocatalytic degradation of reactive dyes in a $\mathrm{TiO} 2$ slurry reactor. J. Photochem. Photobiol. A, 2002. 149: p. 147-154.

18. A. F. Alkaim, T. A. Kandiel, F. H. Hussein, R. Dillert, and D. W. Bahnemann, Enhancing the photocatalytic activity of $\mathrm{TiO} 2$ by $\mathrm{pH}$ control: a case study for the degradation of EDTA. Catal. Sci. Technol., 2013. 3(1): p. 3216-3222.

19. B. Pare, S. B. Jonnalagadda, H. Tomar, P. Singh, and V. W. Bhagwat, $\mathrm{ZnO}$ assisted photocatalytic degradation of acridine orange in aqueous solution using visible irradiation. Desalination, 2008. 232(1-3): p. 80-90.

20. N. Daneshvar, D. Salari, and A. R. Khataee, Photocatalytic degradation of azo dye acid red 14 in water on $\mathrm{ZnO}$ as an alternative catalyst to TiO2. J. Photochem. Photobiol. A, 2004. 162(2â€“3): p. 317-322.

21. Miao J, J.Z., Lu HB, Habibi D and Zhang L, , Heterogeneous photocatalytic degradation of mordant black 11 with $\mathrm{ZnO}$ nanoparticles under UV-Vis light. Journal of the Taiwan Institute of Chemical Engineers, 2014. 45: p. 1636-1641

22. AF Alkaim, T.K., FH Hussein, R Dillert, DW Bahnemann, Solvent-free hydrothermal synthesis of anatase $\mathrm{TiO} 2$ nanoparticles with enhanced photocatalytic hydrogen production activity. Applied zatalysis A: General 2013. 466 p. 32-37. 